Ferrata Storti Foundation

\title{
Utility of positron emission tomography-computed tomography in patients with chronic lymphocytic leukemia following B-cell receptor pathway inhibitor therapy
}

Haematologica 2019

Volume 104(11):2258-2264

\section{Correspondence:}

ANTHONY R. MATO

matoa@mskcc.org

Received: September 28, 2018.

Accepted: March 20, 2019.

Pre-published: March 28, 2019.

doi:10.3324/haematol.2018.207068

Check the online version for the most updated information on this article, online supplements, and information on authorship \& disclosures: www.haematologica.org/content/104/11/2258

(C)2019 Ferrata Storti Foundation

Material published in Haematologica is covered by copyright. All rights are reserved to the Ferrata Storti Foundation. Use of published material is allowed under the following terms and conditions:

https://creativecommons.org/licenses/by-nc/4.0/legalcode. Copies of published material are allowed for personal or internal use. Sharing published material for non-commercial purposes is subject to the following conditions:

https://creativecommons.org/licenses/by-nc/4.0/legalcode, sect. 3. Reproducing and sharing published material for commercial purposes is not allowed without permission in writing from the publisher.
Anthony R. Mato, ${ }^{1}$ William G. Wierda, ${ }^{2}$ Matthew S. Davids, ${ }^{3}$ Bruce D. Cheson, ${ }^{4}$ Steven E. Coutre, ${ }^{5}$ Michael Choi, ${ }^{6}$ Richard R. Furman, ${ }^{7}$ Leonard Heffner, ${ }^{8}$ Paul M. Barr, ${ }^{9}$ Herbert Eradat, ${ }^{10}$ Sharanya M. Ford, ${ }^{11}$ Lang Zhou, ${ }^{11}$ Maria Verdugo, ${ }^{11}$ Rod A. Humerickhouse, ${ }^{11}$ Jalaja Potluri ${ }^{11}$ and John C. Byrd ${ }^{12}$

${ }^{1}$ CLL Program, Leukemia Service, Division of Hematologic Oncology, Memorial Sloan Kettering Cancer Center, New York, NY; '2University of Texas MD Anderson Cancer Center, Houston, TX; ${ }^{3}$ Dana-Farber Cancer Institute, Boston, MA; ${ }^{4}$ Georgetown University Hospital, Washington, DC; ${ }^{5}$ Stanford Cancer Center, Stanford University School of Medicine, Stanford, CA; ${ }^{6}$ UCSD Moores Cancer Center, San Diego, CA; ${ }^{7}$ Weill Cornell Medicine, New York, NY; ${ }^{8}$ Emory University School of Medicine, Atlanta, GA; ' Wilmot Cancer Institute, University of Rochester Medical Center, Rochester, NY; ${ }^{10}$ University of California Los Angeles, Los Angeles, CA; ${ }^{11} \mathrm{AbbVie}$ Inc. North Chicago, IL and ${ }^{12}$ The Ohio State University, Columbus, OH, USA

\section{ABSTRACT}

The utility of positron emission tomography-computed tomography (PET-CT) in distinguishing Richter's transformation versus chronic lymphocytic leukemia (CLL) progression after ibrutinib and/or idelalisib was assessed in a post hoc analysis of a phase II study of venetoclax. Patients underwent PET-CT at screening and were not enrolled/treated if Richter's transformation was confirmed pathologically. Of 167 patients screened, 57 met criteria for biopsy after PET-CT. Of 35 patients who underwent biopsy, eight had Richter's transformation, two had another malignancy, and 25 had CLL. A PET-CT maximum standardized uptake value (SUVmax) $\geq 10$ had $71 \%$ sensitivity and $50 \%$ specificity for detecting Richter's transformation [Odds Ratio (OR): 2.5, 95\%CI: 0.4-15; $P=0.318$ ]. Response rate to venetoclax was similar for screening SUVmax $<10$ versus $\geq 10(65 \%$ vs. $62 \%)$ ( $n=127$ enrolled), though median progression-free survival was longer at $<10$ months (24.7 vs. 15.4 months; $P=0.0335$ ). Six patients developed Richter's transformation on venetoclax, of whom two had screening biopsy demonstrating CLL (others did not have a biopsy) and five had screening SUVmax $<10$. We have defined the test characteristics for PET-CT to distinguish progression of CLL as compared to Richter's transformation when biopsied in patients treated with B-cell receptor signaling pathway inhibitors. Overall diminished sensitivity and specificity as compared to prior reports of patients treated with chemotherapy/ chemoimmunotherapy suggest it has diminished ability to discriminate these two diagnoses using a SUVmax $\geq 10$ cutoff. This cutoff did not identify venetoclax-treated patients with an inferior response but may be predictive of inferior progression-free survival. (Registered at clinicaltrials.gov identifier: 02141282)

\section{Introduction}

Chronic lymphocytic leukemia (CLL) is an indolent, low-grade B-cell lymphoproliferative disorder, which can undergo Richter's transformation (RT) to a diffuse large B-cell lymphoma (DLBCL) ${ }^{1}$ or Hodgkin lymphoma. The prognosis of RT is extremely poor, with a median survival of about eight months. ${ }^{2.5}$ Clinical signs and symptoms associated with RT are non-specific and include rapid clinical deterioration. ${ }^{6}$ Potential factors that may predict RT include mutational status, such as TP53 
dysfunction (including 17p deletion and/or TP53 mutation), advanced stage disease, disease burden, and therapeutic regimen. ${ }^{1,6,7}$

Chronic lymphocytic leukemia generally exhibits low levels of 18-F-fluorodeoxyglucose (FDG) uptake on positron emission tomography-computed tomography (PET-CT), compared to aggressive lymphomas and transformed lymphomas. 8 This difference suggests that PETCT would be a useful tool for distinguishing aggressive versus indolent lymphomas and for selection of nodes for transformation confirmation biopsy. Various studies examining CLL and RT provide a considerable amount of data to inform the use of PET-CT imaging as a tool for distinguishing RT from CLL after chemoimmunotherapy, including standardized uptake value (SUVmax) cutoff thresholds. ${ }^{3,9,10}$ Recent studies have emphasized the unfavorable prognosis for patients with RT following treatment with targeted agents, such as the B-cell receptor signaling pathway inhibitors (BCRi) ibrutinib or idelalisib. 11,12 $^{2}$ Patients who progress after BCRi therapy often demonstrate highly proliferative CLL, even in the absence of a RT, possibly relating to biological differences required for rapid disease progression after $\mathrm{BCRi}$, and thus requiring re-evaluation of PET-CT SUVmax cutoffs for distinguishing RT from CLL.

Venetoclax is a selective, orally bioavailable small-molecule BCL-2 inhibitor that has been approved for patients with previously-treated CLL del(17)(p13.1) and for those without del(17)(p13.1) or TP53 mutation who have not responded to chemoimmunotherapy and BCR-inhibitor therapy. ${ }^{13,14}$ Given the promising efficacy of venetoclax across a broad range of patients with relapsed/refractory CLL, ${ }^{15,16}$ an open-label, phase II study was conducted to evaluate venetoclax monotherapy in patients with CLL refractory to or progressed after discontinuation of ibrutinib or idelalisib. Per protocol, all consenting patients were required to undergo PET-CT imaging at screening, with FDG-avid lymph node biopsy in patients with a suspicion of RT. Here we describe a post hoc analysis to determine if PET-CT and/or patient characteristics were able to differentiate RT versus CLL progression for patients who discontinued BCRi. We also report on the incidence of RT on venetoclax in these patients and how pre-venetoclax PETCT SUVmax may predict clinical outcomes.

\section{Methods}

\section{Study design and overview}

Data from a phase II, open-label, multicenter trial (clinicaltrials.gov identifier: 02141282) of venetoclax monotherapy enrolled patients with CLL relapsed/refractory following ibrutinib or idelalisib were analyzed post hoc. ${ }^{17}$ At each participating site, the institutional review board approved the study protocol and amendments. Study activities were conducted in accordance with ethical principles established in the Declaration of Helsinki and the International Conference on Harmonization Guideline for Good Clinical Practice. All patients provided written informed consent.

\section{Patients}

Adults with CLL refractory to or progressed after discontinuation of ibrutinib or idelalisib who required therapy according to 2008 International Workshop on Chronic Lymphocytic Leukemia (iwCLL) criteria $^{18}$ were eligible. Included patients had Eastern
Cooperative Oncology Group performance score of $\leq 2$, adequate bone marrow function (absolute neutrophil count $\geq 1 \times 10^{9} / \mathrm{L}$, platelet count $\geq 30 \times 10^{9} / \mathrm{L}$, hemoglobin $\geq 8 \mathrm{~g} / \mathrm{dL}$ ), and creatinine clearance $\geq 50 \mathrm{~mL} /$ minute. Patients were excluded if RT was confirmed on biopsy (see details below), and if they had active and uncontrolled autoimmune cytopenias, unresolved toxicities from prior therapy, or allogeneic stem cell transplantation within one year of study entry. ${ }^{17}$

\section{Venetoclax treatment}

Venetoclax was administered orally once daily beginning no sooner than three days after the last prior therapy. Patients received $20 \mathrm{mg}$ daily for one week, followed by a weekly dose increase to reach the final dose of $400 \mathrm{mg}$ daily by week $5 .^{17}$

\section{Assessments}

Screening - patients who signed informed consent, had at least one screening assessment; in case of failed screening they did not proceed on the study. PET-CT imaging within 28 days prior to venetoclax administration was performed on all patients; results were locally reviewed and the node with highest SUV that met protocol criteria was chosen to have either excisional or core biopsy. Based on published literature, ${ }^{3,9,19}$ and as per the study protocol, biopsy of the suspicious area was mandatory if FDG uptake on PET-CT scan was above SUVmax $\geq 10$, or for patients with CD38 positive, ZAP-70 positive, TP53 disrupted or IGHV unmutated CLL with SUVmax 4-10 with at least one of the following: Bsymptoms, lymph node $>5 \mathrm{~cm}$, and/or lactate dehydrogenase (LDH) elevation. Additional screening procedures and on-study assessments are described in the Online Supplementary Appendix.

\section{Statistical analysis}

Using June $30^{\text {th }} 2017$ as the data cutoff, this post hoc analysis was conducted to evaluate PET-CT characteristics (lymph node SUVmax $\geq 5$ and SUVmax $\geq 10$ ) and patients' clinical features that may differentiate RT from CLL progression for patients who were screening for this study following discontinuation of prior BCRi therapy. ${ }^{3,9}$ Descriptive statistics were used to describe PET-CT test characteristics and patients' clinical features. Logistic regression analyses were performed to evaluate if biopsy following PET-CT could differentiate RT post-BCRi therapy during screening. Additional available data on clinical/laboratory parameters for CD38, ZAP70, TP53 and IGHV mutation status, LDH, beta-2 ( $\beta 2)$ microglobulin, and tumor size by logistic regression were also evaluated to determine if any of these patient features may distinguish RT from CLL in this patient population. Based on statistical experience, the small sample size, and uniform direction for all analyses for clinical applicability, sensitivity, specificity, positive predictive value (PPV), negative predictive value (NPV), and area under the receiver operator characteristic (ROC) curve were calculated. The following definitions were used.

Sensitivity: number of patients with true positive divided by observed positive;

Specificity: number of patients with true negative divided by observed negative;

PPV: number of patients with true positive divided by estimated positive;

NPV: number of patients with true negative divided by estimated negative.

Statistical analyses were evaluated for all patients who enrolled and received at least one dose of venetoclax. Overall response rate (ORR) was calculated with a 95\% confidence interval based on binomial distribution. Kaplan-Meier methods were used for timeto-event analyses. Differences in outcomes on venetoclax were stratified based on screening SUVmax cutoff of 10. SAS software 
Table 1. Positron emission tomography-computed tomography findings and baseline characteristics for screened patients.

\begin{tabular}{|c|c|}
\hline & $\begin{array}{c}\text { Screened patients } \\
\qquad=167\end{array}$ \\
\hline \multicolumn{2}{|l|}{ PET-CT findings at screening } \\
\hline Number of FDG-avid nodes, ${ }^{*}$ median (range) & $5(1-12)$ \\
\hline SUVmax, median (range) & $5(0-73)$ \\
\hline SUVmax $\geq 10$ & $25(15)$ \\
\hline \multicolumn{2}{|l|}{ Baseline patient characteristics } \\
\hline Age, median (range), years & $67(28-85)$ \\
\hline N. of prior therapies, median (range) & $4(1-15)$ \\
\hline Ibrutinib, n (\%) & $104(62)$ \\
\hline Idelalisib, n (\%) & $55(33)$ \\
\hline Ibrutinib and idelalisib, $\mathrm{n}(\%)$ & $37(22)$ \\
\hline Purine analogs, $n(\%)$ & $104(62)$ \\
\hline Rituximab/other monoclonal antibodies, n (\%) & $146(87) / 56(34)$ \\
\hline Bendamustine/other alkylating agents, n (\%) & $75(45) / 124(74)$ \\
\hline Bulky nodes $\geq 5$ cm, n (\%) & $69(41)$ \\
\hline Bulky nodes $\geq 10$ cm, n (\%) & $17(10)$ \\
\hline$\beta-2$ microglobulin, ${ }^{\ddagger}$ median (range), mg/L & $3.4(0-59.6)$ \\
\hline Lactate dehydrogenase above the upper limit of normal, ${ }^{\ddagger} \mathrm{n} / \mathrm{N}(\%)$ & $111 / 164(68)$ \\
\hline \multicolumn{2}{|l|}{ Prognostic factors, ${ }^{\ddagger} \mathrm{n} / \mathrm{N}(\%)$} \\
\hline Unmutated IGHV & $93 / 118(79)$ \\
\hline $\operatorname{del}(17 p)$ & $69 / 166(42)$ \\
\hline $\operatorname{del}(11 q)$ & $56 / 165(34)$ \\
\hline TP53 mutation & 48/161 (30) \\
\hline CD38 positive & $70 / 155$ (45) \\
\hline ZAP-70 positive & $45 / 125(36)$ \\
\hline
\end{tabular}

PET-CT: positron emission tomography-computed tomography; FDG: 18-F-fluorodeoxyglucose, SUVmax: maximum standardized uptake value of FDG. *PET-avid defined as SUV $>3$ per nuclear medicine ranges provided by participating institutions. \$Site reported data; presented for all patients with available data. $\mathrm{N} / \mathrm{n}$ : number.

(SAS Institute Inc., Cary, NC) was used to generate all statistical summaries. Unless otherwise stated, statistical analyses were 2sided with $P \leq 0.05$ considered significant.

\section{Results}

\section{Patients' characteristics}

A total of 167 BCRi-failure patients were screened for this study (Online Supplementary Figure S1). There were 40 patients who were screened but did not proceed in the study: $:^{20} 27$ failed to meet inclusion criteria per study protocol [most commonly due to inadequate liver or renal function ( $\mathrm{n}=9$ ), biopsy confirmed RT ( $\mathrm{n}=7)$, and inadequate bone marrow function $(\mathrm{n}=5)]$, nine withdrew consent (for different reasons), and four did not participate for other reasons [investigator decision, second primary malignancy discovered on screening (not RT), spleen biopsy required to assess for RT but not recommended by investigator, congestive heart failure ( $\mathrm{n}=1$ each)]. Demographics and baseline characteristics for all screened patients are presented in Table 1. Patients had received a median of four prior therapies (range: $1-15$ ), $42 \%$ of patients with available data had chromosome 17p deletion (del[17p]), 30\% had TP53 mutation, and 79\% had unmutated IGHV.

\section{Positron emission tomography-computed tomography imaging and biopsy at screening}

Of the 167 screened patients, $84(50 \%)$ had a lymph node with SUVmax $\geq 5$ and $25(15 \%)$ patients had SUVmax $\geq 10$ (Table 1), ${ }^{3,9}$ Following PET-CT imaging, 57 patients met protocol criteria for the required core biopsy of the FDG-avid lymph node to evaluate for RT, of whom 22 failed screening for other reasons and did not have a biopsy. (Other reasons for screen failure were heterogeneous and have been previously reported ${ }^{20}$ ) (Online Supplementary Figure S1). Of the remaining 35 patients who underwent biopsy, 19 had SUVmax $\geq 10$ and 16 had SUVmax $<10$ with other high risk clinical/laboratory features that mandated biopsy. Additionally, one patient had an abnormal CT result prior to study-related procedures which led to biopsy of a node during screening (patient did not have PET-CT at screening). Eight patients had RT: $22 \%$ of all patients who were biopsied $(n=36)$ and $4.8 \%$ of all 167 patients progressing after BCRi screened for this clinical trial (all DLBCL). These eight patients were not enrolled on the clinical trial (Table 2), three failed screening due to other reasons including other malignancies (metastatic anal cancer and neuroendocrine tumor), and 25 patients had a biopsy demonstrating CLL without transformation and were subsequently enrolled on study. Of the eight patients with biopsy-confirmed RT, five had 
Table 2. Characteristics of patients with biopsy-confirmed Richter's transformation at screening.

\begin{tabular}{|c|c|c|c|c|c|c|c|c|c|}
\hline & & & & & Patients & & & & Summary \\
\hline & 1 & 2 & 3 & 4 & 5 & 6 & 7 & 8 & \\
\hline Age ${ }^{\dagger}$ years & 54 & 81 & 63 & 76 & 84 & 81 & 72 & 68 & $74(54-84)$ \\
\hline $\operatorname{Sex}^{\ddagger}$ & Female & Male & Male & Male & Male & Male & Male & Male & $7(88)$ \\
\hline Time from diagnosis, ${ }^{\dagger}$ years & 6.7 & 6.6 & 3.1 & 3.3 & 6.2 & 14.2 & 16 & $\mathrm{~N} / \mathrm{A}$ & $6.6(3.2-16)$ \\
\hline Prior therapies, ${ }^{\dagger} \mathrm{n}$ & 3 & 8 & 2 & 3 & 3 & 12 & 9 & 3 & $3(2-12)$ \\
\hline $\operatorname{del}(17 \mathrm{p}) /$ TP53 mutation ${ }^{\ddagger}$ & Yes & No & Yes & Yes & Yes & No & Yes & Yes & $5(63)$ \\
\hline Screening PET-CT SUVmax ${ }^{\dagger}$ & 12 & 19 & 12 & 11 & 22 & 2 & 0.5 & N/A* & $12(0.5-22)$ \\
\hline Largest node at baseline, ${ }^{\dagger} \mathrm{cm}$ & 3 & 8 & 3 & 7 & $\mathrm{~N} / \mathrm{A}$ & $\mathrm{N} / \mathrm{A}$ & N/A & 4 & $4(3-8)$ \\
\hline$\beta 2$ microglobulin, ${ }^{\dagger} \mathrm{mg} / \mathrm{L}$ & $\mathrm{n} / \mathrm{A}$ & 3.7 & 1.6 & 5.9 & 3.5 & N/A & N/A & N/A & $3.6(1.6-5.9)$ \\
\hline $\mathrm{ALC}, \mathrm{x} 10^{9} / \mathrm{L}^{\dagger}$ & 1.5 & 30.9 & 21.4 & 1.3 & 30.4 & 1.1 & 3.5 & 160 & $12.4(1.1-160)$ \\
\hline
\end{tabular}

del(17p): chromosome 17p deletion; PET-CT: positron emission tomography-computed tomography; SUVmax: maximum standardized uptake value of 18-F-fluorodeoxyglucose; ALC: absolute lymphocyte count; N/A: data not available. †Summary column describes the median (range) across the eight patients. $¥$ Summary column describes the number (n) (\%) across the eight patients. *Patient had an abnormal CT SCAN prior to screening for study, which led to a biopsy to confirm Richter's transformation. PET-CT was not performed but the patient was excluded based on biopsy findings.

A

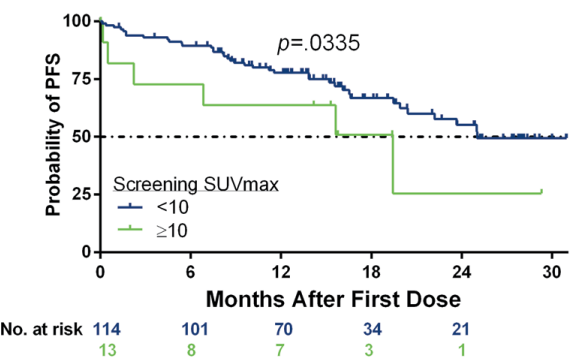

C

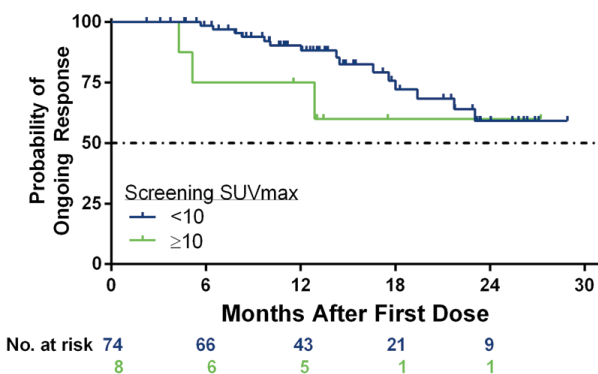

B

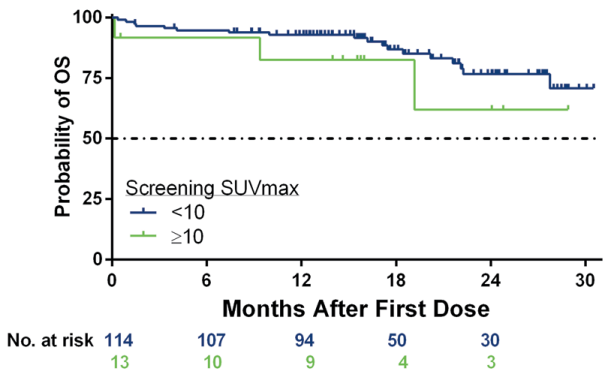

Figure 1. Outcomes on venetoclax stratified by screening maximum standardized uptake value of 18-F-fluorodeoxyglucose (SUVmax) by positron emission tomographycomputed tomography imaging. Shown are the investigator-assessed (A) progressionfree survival (PFS), and (B) overall survival (OS) on venetoclax as assessed by the investigator for patients with screening SUVmax $<10(n=114)$ and SUVmax $\geq 10 \quad(n=13)$. (C) Investigator-assessed duration of response is shown for responders on venetoclax strat ified by screening SUVmax $<10(n=74)$ and SUVmax $\geq 10(n=8)$. Number of patients at risk for the event at each time point is shown below each curve. Tick marks represent censored data. n: number.
SUVmax $\geq 10$, two had SUVmax $<10$ with other factors that required biopsy per protocol, and one did not have PET-CT at screening and thus was not included in the following sensitivity/specificity analysis. Fourteen patients with SUVmax $\geq 10$ had biopsy demonstrating CLL without evidence of RT. Of the 14 patients with SUVmax $\geq 10$ with biopsy demonstrating CLL, nine patients were off BCRi [7 off ibrutinib and 2 off idelalisib with median time off therapy of 0.8 months (range: $0.1-18$ )] at the time of PET-CT, and five patients were on a BCRi at the time of PET-CT (3 on ibrutinib, 2 on idelalisib). There was no difference between the median SUVmax in patients on or off BCRi at time of PET-CT [5 (range: 0.5-28) vs. 5 (range: 073), respectively].

In a prior analysis, Michallet et al. showed that SUVmax $>10$ was the most effective cutoff value to identify $\mathrm{RT}$ in patients largely treated with chemotherapy or chemoimmunotherapy. ${ }^{10}$ Given this, we also evaluated this cutoff in the post-BCRi setting. In the current analysis, screening SUVmax $\geq 10$ had $71 \%$ sensitivity, $50 \%$ specificity, $26 \%$ PPV, and 88\% NPV for detection of biopsy-confirmed RT versus CLL progression in patients post-BCRi therapy [odds ratio: 2.5 (0.4-15); $P=0.318)$ ] (Table 3). The ROC area for SUVmax $\geq 10$ was $61 \%$. Other reports have shown that SUVmax $\geq 5$ provided high sensitivity and specificity to distinguish RT in patients treated with chemotherapy or chemoimmunotherapy., In BCRi-exposed patients, we observed no difference in sensitivity with SUVmax $\geq 5$ (71\%), with decreased specificity for identifying RT (4\%) versus what we report for SUVmax $\geq 10$. SUVmax cutoff points of $\geq 11$ and $\geq 12$ were also evaluated, although the sensitivity remained the same or decreased compared 
Table 3. Detection of biopsy-confirmed Richter's transformation (RT) versus chronic lymphocytic leukemia (CLL) progression based on positron emission tomography-computed tomography and clinical factors.

\begin{tabular}{|c|c|c|c|c|c|c|}
\hline & & & & gressic & & \\
\hline & Sensitivity & Specificity & PPV & NPV & ROC area & $\begin{array}{c}\text { Odds ratio }[95 \% \text { Cl] } \\
\text { logistic } \boldsymbol{P}\end{array}$ \\
\hline PET-CT SUVmax $<10 / \geq 10$ & $71 \%$ & $50 \%$ & $26 \%$ & $88 \%$ & $61 \%$ & $2.5[0.4-15], P=0.318$ \\
\hline PET-CT SUVmax <5/ $\geq 5$ & $71 \%$ & $4 \%$ & $16 \%$ & $33 \%$ & $63 \%$ & $0.09[0.01-1.2], P=0.071$ \\
\hline PET-CT SUVmax $<11 / \geq 11$ & $71 \%$ & $61 \%$ & $31 \%$ & $89 \%$ & $66 \%$ & $3.8[0.6-24], P=0.143$ \\
\hline PET-CT SUVmax $<12 / \geq 12$ & $57 \%$ & $68 \%$ & $31 \%$ & $86 \%$ & $63 \%$ & $2.8[0.5-15], P=0.231$ \\
\hline Lactate dehydrogenase $\leq />$ ULN & $83 \%$ & $29 \%$ & $20 \%$ & $89 \%$ & $56 \%$ & $2[0.2-20], P=0.554$ \\
\hline SPD at baseline & $67 \%$ & $48 \%$ & $24 \%$ & $86 \%$ & $59 \%$ & $1[1-1], P=0.244$ \\
\hline TP53* mutated/unmutated & $60 \%$ & $63 \%$ & $25 \%$ & $88 \%$ & $61 \%$ & $2.5[0.35-18], P=0.362$ \\
\hline IGHV * mutated/unmutated & $25 \%$ & $86 \%$ & $25 \%$ & $86 \%$ & $56 \%$ & $2.1[0.16-28], P=0.569$ \\
\hline CD-38* positive/negative & $25 \%$ & $40 \%$ & $6 \%$ & $77 \%$ & $68 \%$ & $0.22[0.02-2.5], P=0.220$ \\
\hline ZAP-70* positive/negative & $50 \%$ & $23 \%$ & $9 \%$ & $75 \%$ & $64 \%$ & $0.3[0.01-6.4], P=0.440$ \\
\hline$\beta 2$ microglobulin * $</ \geq 3 \mathrm{mg} / \mathrm{L}$ & $75 \%$ & $25 \%$ & $20 \%$ & $80 \%$ & $50 \%$ & $1[0.08-13], P=1.0$ \\
\hline
\end{tabular}

PPV: positive predictive value; NPV: negative predictive value; ROC: Receiver Operator Characteristic; PET-CT: positron emission tomography-computed tomography; SUVmax maximum standardized uptake value of 18-F-fluorodeoxyglucose; ULN: upper limit of the normal range; SPD: sum products of the greatest transverse diameters (tumor size) * Site-reported data. The logistic regression analyses only included patients with available data.

with SUVmax $\geq 10$. Other baseline clinical/prognostic features were evaluated, including above normal LDH and $\beta 2$-microglobulin levels (Table 3).

\section{Outcomes on venetoclax}

At the time of analysis, patients enrolled on study had been on venetoclax for a median of 10.3 months (range: 0.1-26 months). Falchi et al. had previously reported that patients with SUVmax $\geq 10$ had an inferior survival on chemotherapy/chemoimmunotherapy; ${ }^{3}$ therefore, we evaluated outcomes of venetoclax using this cutoff of screening SUVmax. The ORR on venetoclax was $65 \%(82$ of 127) for all enrolled patients, and was similar when stratified by screening PET-CT SUVmax $<10(65 \%, 74$ of $114)$ versus $\geq 10(62 \%$, 8 of 13$)(P=0.81)$. Median progression-free survival (PFS) was longer for venetoclax-treated patients with screening PET-CT SUVmax $<10$ versus $\geq 10$ [24.7 months (95\%CI: 20.1, -) vs. 15.4 months [95\% CI: 0.4, -]; $P=0.0335$ ), with Kaplan-Meier estimates at 12 months of $79 \%$ (95\% CI: $69 \%, 85 \%)$ and $58 \%(95 \% \mathrm{CI}$ : $27 \%, 80 \%$ ), respectively (Figure 1A). The median time on study, including follow up, was 13.8 months (range: 0.0331 months). Median overall survival had not been reached at the time of analysis, though 12 -month estimates for patients with SUVmax $<10$ versus $\geq 10$ were $94 \%$ (95\%CI: $87 \%, 97 \%)$ and $76 \%(95 \% \mathrm{CI}: 43,92 \%)$, respectively $(P=0.061)$ (Figure 1B). For patients who responded to venetoclax, the median duration of response had not been reached at the time of analysis, and 12-month estimates for patients with SUVmax $<10$ versus $\geq 10$ were $90 \%$ (95\% CI: $80 \%, 96 \%)$ and $75 \%(95 \%$ CI: $32 \%, 93 \%)$, respectively $(P=0.17)$ (Figure $1 \mathrm{C})$. In an intent-to-treat analysis, $29 \%$ (33 of 114) of patients with screening SUVmax $<10$ achieved minimal residual disease (MRD) negativity in peripheral blood, with 19 of these patients achieving this outcome by week 24. For patients with screening SUVmax $\geq 10,23 \%$ ( 3 of 13 ) achieved MRD negativity in peripheral blood, with one who had this outcome by week 24 . Of three patients with screening SUVmax $\geq 10$ who achieved MRD negativity on veneto-

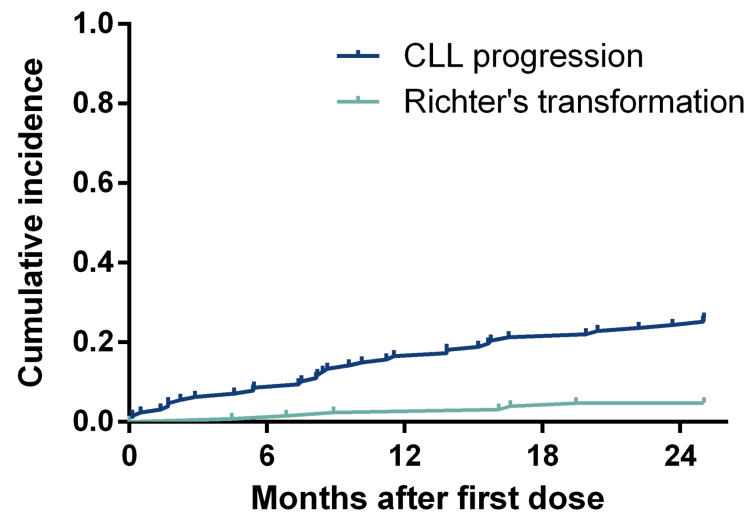

Figure 2. Time to chronic lymphocytic leukemia (CLL) progression or Richter's transformation (RT) on venetoclax. Shown is the cumulative incidence of CLL progression or Richter's transformation on venetoclax. Thirty-three patients discontinued venetoclax due to CLL progression and six due to biopsy-confirmed RT following both imaging and clinical changes. Median time to CLL progression was 8.5 months (range: 0.1-28 months) and to RT was 12.8 months (range: 4.4-19.7 months). Tick marks represent patients with events.

clax, two are still on study and receiving treatment, and one patient discontinued venetoclax and later died due to squamous cell carcinoma of head and neck. There was no statistical difference in the rate of MRD-negativity in blood based on screening SUVmax $\geq 10$ versus $<10$ $(P=0.83)$.

Sixty-two (49\%) patients discontinued venetoclax (Online Supplementary Figure S1), with a median time to discontinuation for any reason of 8.6 months (range: 0.1 28). Thirty-three patients $(26 \%)$ discontinued venetoclax due to CLL progression and six $(4.7 \%)$ due to biopsy-confirmed RT following clinical findings and radiographic changes (Table 4). The median time to CLL progression was 8.5 months (range: 0.1-28 months) and to RT was 12.8 months (range: 4.4-19.7 months) (Figure 2). Of six 
Table 4. Characteristics of patients who underwent Richter's transformation on venetoclax.

\begin{tabular}{|c|c|c|c|c|c|c|c|}
\hline & & & Patients & & & & Summary \\
\hline & 1 & 2 & 3 & 4 & 5 & 6 & \\
\hline Age,$^{\dagger}$ years & 56 & 65 & 58 & 63 & 65 & 66 & $64(56-66)$ \\
\hline $\operatorname{Sex}^{ \pm}$ & Male & Female & Female & Male & Female & Male & $3(50)$ \\
\hline Time from diagnosis, ${ }^{\dagger}$ years & 12 & 12 & 9 & 6 & 18 & 20 & $12(6-20)$ \\
\hline Prior therapies, ${ }^{\dagger} \mathrm{n}$ & 5 & 9 & 9 & 2 & 6 & 5 & $5.5(2-9)$ \\
\hline $\operatorname{del}(17 p) / T P 53$ mutation & Yes & Yes & Yes & No & No & Yes & $4(67)$ \\
\hline Screening PET-CT SUVmax ${ }^{\dagger}$ & 11 & 8 & 5 & $<3$ & 6 & $<3$ & $7(<3-11)$ \\
\hline Largest node at baseline, ${ }^{\dagger} \mathrm{cm}$ & 4.8 & 10.8 & 2.5 & 7.2 & 3.1 & 8.6 & $4.8(2.5-10.8)$ \\
\hline$\beta-2$ microglobulin, ${ }^{\dagger} \mathrm{mg} / \mathrm{L}$ & 2.5 & 5.7 & 2.8 & 6.8 & $\mathrm{~N} / \mathrm{A}$ & 2.8 & $2.8(2.5-6.8)$ \\
\hline $\mathrm{ALC}, \mathrm{x} 10^{9} / \mathrm{L}^{\dagger}$ & 20 & 2 & 71 & 6 & 406 & .9 & $13(0.9-406)$ \\
\hline Best response on venetoclax & PR & SD & PR & PR & $\mathrm{CR}^{*}$ & PR & $83 \%$ ORR \\
\hline Time to RT, ${ }^{\dagger}$ weeks & 30 & 19 & 87 & 68 & 72 & 71 & $69.5(19-87)$ \\
\hline
\end{tabular}

del(17p): chromosome 17p deletion; PET-CT: positron emission tomography-computed tomography; SUVmax: maximum standardized uptake value of 18-F-fluorodeoxyglucose; N/A: data not available; ALC: absolute lymphocyte count; PR: partial remission; SD: stable disease; CR: complete remission; ORR: overall response rate (CR+PR); RT: Richter's transformation. *Patient had PR at weeks 8 and 24 and then CR at week 60 . $\uparrow$ Summary column describes the median (range) across the six patients. $¥$ Summary column describes the number (n) (\%) across the six patients.

patients who discontinued venetoclax due to a relapse with RT, two met criteria for biopsy and underwent biopsy at screening. Both biopsies were negative for RT, though one patient had prior RT and the other had prior DLBCL both more than two years prior to enrollment. Five of the six patients who developed RT on venetoclax had screening SUVmax on PET $<10$ and screening SUVmax for all six patients with RT was not statistically different from other enrolled patients $(P=0.24)$.

\section{Discussion}

The diagnosis and management of RT remain challenging due to rapid progression and refractoriness to chemotherapy and targeted therapies. ${ }^{3}$ Evaluation of patients following chemotherapy or chemoimmunotherapy showed that PET-CT imaging is helpful to detect RT and identify sites to target for biopsy, ${ }^{3,9,10}$ with SUVmax of $\geq 10$ identified as an optimal threshold for distinguishing RT versus CLL progression, ${ }^{10}$ and associated with poor survival, independently of RT diagnosis. ${ }^{3}$

Newer treatments, including BCRi or BCL-2 inhibitors, are highly active for patients with relapsed/refractory CLL $^{15,16,21,22}$ and have begun to supplant the use of chemotherapy. Data reported here represent the largest series of prospective PET-CT scans performed based on predetermined criteria in patients following discontinuation of either ibrutinib or idelalisib. Of 167 patients who were screened for this phase II study, 57 met protocol criteria for a biopsy to evaluate for RT following PET-CT imaging, and RT was confirmed for $4.8 \%$ of all screened patients. Five of these patients had SUVmax $\geq 10$ while the other two had SUVmax $<10$ with other factors associated with RT (e.g. LDH elevation or B symptoms). Based on this analysis, we report the test characteristics for PET-CT with SUVmax $\geq 10$. We observed a lower sensitivity (71\%) and specificity $(50 \%)$ for SUVmax $\geq 10$ in patients who have been exposed to BCRi therapy as compared to prior reports in patients not progressing on $\mathrm{BCRi} .{ }^{10} \mathrm{We}$ emphasize the importance of biopsy to confirm clinical or radiographic suspicion of RT or a secondary malignancy. PETCT should not be used without biopsy to diagnose RT.
Our data suggest that CLL progression following BCRi exposure may be more metabolically active compared to progression following chemoimmunotherapy. In addition, these data suggest no difference in FDG avidity in patients with CLL regardless of whether they were on their BCRi at the time of imaging or not. Only LDH above the upper limit of normal and $\beta-2$ microglobulin $\geq 3 \mathrm{mg} / \mathrm{L}$ had high sensitivity for detection of RT from CLL progression. Limitations of our study are the small sample size in each group of patients on or off BCRi at the time of PET-CT and the fact that other patient features (such as cytogenetics) were not available for all patients, so statistical analyses for those factors only include subsets of the patients with available data. Another limitation of the analysis was that PET-CT was not centrally reviewed and there may be differences in imaging methodologies, number of lymph nodes assessed per study, and interpretations of imaging studies. Though pathology results were also not centrally reviewed, all biopsies were reviewed by hematopathology experts at the study sites, which were major academic institutions. Although there was a relatively small number of patients with RT events included in these analyses, to our knowledge, this dataset is the largest of BCRi-treated patients who had PET-CT and were prospectively evaluated.

For 127 patients who enrolled in the study, no difference was seen in the response rate with venetoclax monotherapy when stratified by screening SUVmax of 10 in whom a biopsy ruled out RT, suggesting that PET-CT performed prior to venetoclax initiation may identify patients with an inferior PFS. We have identified a population of highrisk CLL patients previously treated with BCRi for whom venetoclax monotherapy may result in inferior PFS and in whom venetoclax-based combination regimens should be studied.

Whereas in prior ibrutinib studies, the development of RT appeared to be a relatively early event (median approx. 6 months), ${ }^{23}$ in this study, the development of RT on venetoclax for six patients occurred later (median 12.8 months). This may reflect differences in how individual therapies (BCRi vs. BCL2 inhibitor) affect the CLL clone and/or the patient populations. More likely, the later 
occurrence of RT in the current study suggests that most patients with RT were prospectively identified and excluded from participating in this study utilizing the prespecified criteria for biopsy based on PET-CT imaging and other clinical features. It is also possible that there was a treatment effect of venetoclax, which would be consistent with the results for the DLBCL patients with RT in a previous Phase I study across non-Hodgkin lymphomas. ${ }^{24}$ Venetoclax is currently being studied in CLL patients with RT (clinicaltrials.gov identifier: 03054896).

The primary role of PET-CT in patients with CLL is to identify the most appropriate target for biopsy to assess for the presence of RT or other malignancy. Overall, our data suggest that CLL progression may be more metabolically active on PET-CT following BCRi failure as compared to chemoimmunotherapy. PET-CT SUVmax cutoff of 10 was able to distinguish suspected RT from CLL progression at screening with a sensitivity of $71 \%$ and specificity of $50 \%$, test characteristics which are modest compared to those reported in prior studies with chemotherapy or CIT-treated patients. ${ }^{10}$ SUVmax cutoff of 10 at baseline also did not predict the development of RT in patients on venetoclax. Clinical features, including elevated $\beta-2$ microglobulin and LDH may provide additional information when considering biopsy to confirm RT. Future analyses including assessment of additional genetic fac- tors, BCR stereotypes, or gene expression could be tested as predictors for the risk of RT following BCRi exposure.

\section{Acknowledgments}

Special thanks to the patients and their families, study coordinators, and support staff.

\section{Funding}

AbbVie and Genentech provided financial support for the study. AbbVie is committed to responsible data sharing regarding the clinical trials we sponsor. This includes access to anonymized, individual and trial-level data (analysis data sets), as well as other information (e.g. protocols and Clinical Study Reports), as long as the trials are not part of an ongoing or planned regulatory submission. This includes requests for clinical trial data for unlicensed products and indications. This clinical trial data can be requested by any qualified researchers who engage in rigorous, independent scientific research, and will be provided following review and approval of a research proposal and Statistical Analysis Plan and execution of a Data Sharing Agreement. Data requests can be submitted at any time and the data will be accessible for 12 months, with possible extensions considered. For more information on the process, or to submit a request, visit the following link: https://www.abbvie.com/our-science/clinical-trials/clinical-trials-data-and-information-sharing/data-and-informationsharing-with-qualified-researchers.html.

\section{References}

1. Rossi D, Cerri M, Capello D, et al. Biological and clinical risk factors of chronic lymphocytic leukaemia transformation to Richter syndrome. Br J Haematol. 2008;142(2):202215.

2. Molica S. A systematic review on Richter syndrome: what is the published evidence? Leuk Lymphoma. 2010;51(3):415-421.

3. Falchi L, Keating MJ, Marom EM, et al. Correlation between FDG/PET, histology, characteristics, and survival in 332 patients with chronic lymphoid leukemia. Blood. 2014;123(18):2783-2790.

4. Tsimberidou AM, Kantarjian HM, Cortes J, et al. Fractionated cyclophosphamide, vincristine, liposomal daunorubicin, and dexamethasone plus rituximab and granulocyte-macrophage-colony stimulating factor (GM-CSF) alternating with methotrexate and cytarabine plus rituximab and GM-CSF in patients with Richter syndrome or fludarabine-refractory chronic lymphocytic leukemia. Cancer. 2003;97(7):1711-1720.

5. Tsimberidou AM, O'Brien SM, Cortes JE, et al. Phase II study of fludarabine, cytarabine (Ara-C), cyclophosphamide, cisplatin and GM-CSF (FACPGM) in patients with Richter's syndrome or refractory lymphoproliferative disorders. Leuk Lymphoma. 2002:43(4):767-772.

6. Rossi D, Gaidano G. Richter syndrome: pathogenesis and management. Semin Oncol. 2016:43(2):311-319.

7. Parikh SA, Rabe KG, Call TG, et al. Diffuse large B-cell lymphoma (Richter syndrome) in patients with chronic lymphocytic leukaemia (CLL): a cohort study of newly diagnosed patients. Br J Haematol. 2013;162(6):774-782.
8. Shaikh F, Janjua A, Van Gestel F, Ahmad A. Richter Transformation of Chronic Lymphocytic Leukemia: A Review of Fluorodeoxyglucose Positron Emission Tomography-Computed Tomography and Molecular Diagnostics. Cureus. 2017; 9(1):e968.

9. Bruzzi JF, Macapinlac H, Tsimberidou AM, et al. Detection of Richter's transformation of chronic lymphocytic leukemia by PET/CT. J Nucl Med. 2006;47(8):1267-1273.

10. Michallet AS, Sesques P, Rabe KG, et al. An 18F-FDG-PET maximum standardized uptake value $>10$ represents a novel valid marker for discerning Richter's Syndrome. Leuk Lymphoma. 2016;57(6):1474-1477

11. Jain P, Keating M, Wierda W, et al Outcomes of patients with chronic lymphocytic leukemia after discontinuing ibrutinib. Blood. 2015;125(13):2062-2067.

12. Maddocks KJ, Ruppert AS, Lozanski G, et al. Etiology of Ibrutinib Therapy Discontinuation and Outcomes in Patients With Chronic Lymphocytic Leukemia. JAMA Oncol. 2015;1(1):80-87.

13. VENCLEXTA [Prescribing Information] AbbVie Inc., North Chicago, IL, USA. Genentech USA Inc., South San Francisco, CA, USA., 2016.

14. VENCLYXTO [Summary of Product Characteristics]. AbbVie Ltd., Maidenhead, United Kingdom., 2016.

15. Roberts AW, Davids MS, Pagel JM, et al. Targeting BCL2 with Venetoclax in Relapsed Chronic Lymphocytic Leukemia. N Engl J Med. 2016;374(4):311-322.

16. Stilgenbauer S, Eichhorst B, Schetelig J, et al. Venetoclax in relapsed/refractory chronic lymphocytic leukemia with $17 p$ deletion: a phase 2, open label, multicenter study. Lancet Oncol. 2016;7(6):768-778.

17. Jones JA, Mato AR, Wierda WG, et al.
Venetoclax for chronic lymphocytic leukaemia progressing after ibrutinib: an interim analysis of a multicentre, open-label, phase 2 trial. Lancet Oncol. 2017;19(1):6575 .

18. Hallek M, Cheson BD, Catovsky D, et al Guidelines for the diagnosis and treatment of chronic lymphocytic leukemia: a report from the International Workshop on Chronic Lymphocytic Leukemia updating the National Cancer Institute-Working Group 1996 guidelines. Blood. 2008; 111(12):5446-5456.

19. Bodet-Milin C, Kraeber-Bodere F, Moreau P, et al. Investigation of FDG-PET/CT imaging to guide biopsies in the detection of histological transformation of indolent lymphoma. Haematologica. 2008:93(3):471-472.

20. Jones JA, Mato AR, Wierda WG, et al. Venetoclax for chronic lymphocytic leukaemia progressing after ibrutinib: an interim analysis of a multicentre, open-label, phase 2 trial. Lancet Oncol. 2018;19(1):65-75

21. Byrd JC, Furman RR, Coutre SE, et al Targeting BTK with ibrutinib in relapsed chronic lymphocytic leukemia. N Engl J Med. 2013;369(1):32-42

22. Furman RR, Sharman JP, Coutre SE, et al. Idelalisib and rituximab in relapsed chronic lymphocytic leukemia. N Engl J Med. 2014;370(11):997-1007.

23. O'Brien S, Jones JA, Coutre SE, et al Ibrutinib for patients with relapsed or refractory chronic lymphocytic leukaemia with $17 p$ deletion (RESONATE-17): a phase 2, open-label, multicentre study. Lancet Oncol. 2016;17(10):1409-1418

24. Davids MS, Roberts AW, Seymour JF, et al Phase I First-in-Human Study of Venetoclax in Patients With Relapsed or Refractory Non-Hodgkin Lymphoma. J Clin Oncol. 2017;35(8):826-833 